\title{
UNBIASED ESTIMATION FOR TWO-PARAMETER EXPONENTIAL DISTRIBUTION UNDER TIME CENSORED SAMPLING
}

Abstract. The problem considered is that of unbiased estimation for a two-parameter exponential distribution under time censored sampling. We obtain a necessary form of an unbiasedly estimable parametric function and prove that there does not exist any unbiased estimator of the parameters and the mean of the distribution. For reliability estimation at a specified time point, we give a necessary and sufficient condition for the existence of an unbiased estimator and suggest an unbiased estimator based on a sufficient statistic in situations where unbiased estimators exist. Unbiased estimation of the variance of an unbiased estimator of reliability is also addressed.

1. Introduction. A two-parameter exponential distribution with real parameters $\mu$ and $\lambda(>0)$, to be denoted hereafter $\operatorname{as} \exp (\mu, \lambda)$ distribution, is defined by the probability function (p.f.)

$$
f(x \mid \mu, \lambda)=\frac{1}{\lambda} e^{-(x-\mu) / \lambda}, \quad x>\mu .
$$

This distribution is widely used as a model in reliability theory.

Suppose the life-length $X$ of an item follows an $\exp (\mu, \lambda)$ distribution with unknown parameters, and consider the problem of unbiased estimation of a real-valued parametric function $\Psi(\mu, \lambda)$. Some particular parametric functions of interest are (i) the location parameter $\mu$; (ii) the scale parameter $\lambda$; (iii) the mean life-length $E(X)=\mu+\lambda$; (iv) the reliability at a

2000 Mathematics Subject Classification: 62N01, 62 N05.

Key words and phrases: mean life, reliability, time censored sampling, two-parameter exponential distribution, unbiased estimation, variance estimation. 
specified time point $t(>0)$, viz.

$$
R(t)=P(X>t)= \begin{cases}1, & t \leq \mu, \\ e^{-(t-\mu) / \lambda}, & t>\mu,\end{cases}
$$

and (v) the variance of an unbiased estimator of $R(t)$ or equivalently $R^{2}(t)$.

For a complete life-testing experiment, the uniformly minimum variance unbiased estimators (UMVUE's) of these parametric functions based on observed life-lengths $X_{1}, \ldots, X_{n}$ of $n$ test items are well known (see Johnson et al. [3, Chapter 19]; Laurent [4]) and are functions of the complete sufficient statistic $\left(X_{(1)}, S\right)$ where $X_{(i)}$ is the life-length of the $i$ th failed item and $S=\sum_{i=2}^{n}\left(X_{(i)}-X_{(1)}\right)$.

In many practical situations, however, a complete life-testing experiment is neither feasible nor desirable due to constraints on time and cost, and estimation is to be based on data obtained from a censored sample wherein the experiment is terminated either after a pre-assigned time $T(>0)$ or when a pre-assigned number of items, say $r(<n)$, have failed. For a censored sample of second type, called a failure censored sample, a complete sufficient statistic is $\left(X_{(1)}, S^{*}\right)$, where $S^{*}=\sum_{i=2}^{r}\left(X_{(i)}-X_{(1)}\right)+(n-r)\left(X_{(r)}-X_{(1)}\right)$, and UMVUE's of parametric functions such as (i) $-(\mathrm{v})$ are obtained as functions of this statistic by suitably modifying the UMVUE's for a complete lifetesting experiment (see Mann et al. [5, Chapter 5]; Basu [2]).

In this paper we address the problem of unbiased estimation for a censored sample of first type, known as a time censored sample, and obtain a necessary form of an unbiasedly estimable parametric function $\Psi(\mu, \lambda)$. We also prove that there does not exist any unbiased estimator of $\mu, \lambda$ and $\mu+\lambda$, and give necessary and sufficient conditions for unbiased estimability of $R(t)$ and $R^{2}(t)$.

In situations where unbiased estimators exist, an unbiased estimator of $R(t)$ based on a sufficient statistic was given in Bartoszewicz [1], and an unbiased estimator of $R^{2}(t)$ can be derived by similar arguments (see Section 3). Bartoszewicz [1], further showed that the sufficient statistic is not, however, complete except for $n=1,2$, and hence an unbiased estimator based on it is not generally unique. In the final section of the paper we suggest alternative unbiased estimators of $R(t)$ and $R^{2}(t)$ based on the sufficient statistic in situations where unbiased estimators exist.

2. Existence of unbiased estimators. For a time censored sample, the data consist of $D$ and $X_{(0)}, X_{(1)}, \ldots, X_{(D)}$, where $D$ is the number of items failed up to pre-assigned time $T(>0)$ out of $n$ test items, $X_{(i)}$ being the life-length of the $i$ th failed item, $1 \leq i \leq D$, and $X_{(0)}=0$. Let $p=R(T)$ and note that $D$ follows a binomial distribution with mean $n q, q=1-p$. 
The joint p.f. of $D$ and $X_{(0)}, X_{(1)}, \ldots, X_{(D)}$ is (see Bartoszewicz [1]) (3)

$$
\begin{aligned}
p\left(d, x_{(0)}, x_{(1)}, \ldots, x_{(d)}\right) & d=0, \\
= & \left\{\begin{aligned}
p^{n}, & r\left(\begin{array}{l}
n \\
d
\end{array}\right) p^{n-d} I(T>\mu) \frac{1}{\lambda^{d}} e^{-\sum_{i=1}^{d}\left(x_{(i)}-\mu\right) / \lambda} I\left(x_{(1)}>\mu\right), \\
1 & \leq d \leq n, x_{(1)}<\cdots<x_{(d)} \leq T
\end{aligned}\right.
\end{aligned}
$$

where $I(A)$ is the indicator function of the set $A$. Clearly a sufficient statistic is $V=\left(D, Z_{D}\right)$ where

$$
Z_{d}= \begin{cases}X_{(d)}, & d=0,1, \\ \left(X_{(1)}, S_{d}=\sum_{i=2}^{d}\left(X_{(i)}-X_{(1)}\right)\right), & 2 \leq d \leq n .\end{cases}
$$

The p.f. of $V$ is given by (see Bartoszewicz [1])

$$
p\left(d, z_{d}\right)= \begin{cases}p^{n}, & d=0, \\
n p^{n-1} q I(T>\mu) p\left(x_{(1)} \mid d\right), & d=1, \\
\left(\begin{array}{l}
n \\
d
\end{array}\right) p^{n-d} q^{d} I(T>\mu) p\left(x_{(1)} \mid d\right) p\left(s_{d} \mid d, x_{(1)}\right), & 2 \leq d \leq n,\end{cases}
$$

where for $d \geq 1$,

$$
\begin{aligned}
& p\left(x_{(1)} \mid d\right)=\text { the conditional p.f. of } X_{(1)} \text { given } D=d \\
& \quad=\frac{d}{\lambda q^{d}} e^{-\left(x_{(1)}-\mu\right) / \lambda}\left[e^{-\left(x_{(1)}-\mu\right) / \lambda}-e^{-(T-\mu) / \lambda}\right]^{d-1}, \quad \mu<x_{(1)} \leq T,
\end{aligned}
$$

and for $d \geq 2$,

$$
\begin{array}{r}
p\left(s_{d} \mid d, x_{(1)}\right)=\text { the conditional p.f. of } S_{d} \text { given } D=d \text { and } X_{(1)}=x_{(1)} \\
=\frac{1}{\Gamma(d-1) \lambda^{d-1}\left(1-e^{-\left(T-x_{(1)}\right) / \lambda}\right)^{d-1}} e^{-s_{d} / \lambda} f_{d}\left(s_{d}, T-x_{(1)}\right), \\
0<s_{d} \leq(d-1)\left(T-x_{(1)}\right),
\end{array}
$$

with

$$
f_{d}(u, w)=\sum_{j=0}^{d-1}(-1)^{j}\left(\begin{array}{c}
d-1 \\
j
\end{array}\right)(u-j w)^{d-2} I(u>j w) .
$$

In view of sufficiency of $V$ it is enough to restrict to estimators based on $V$ to study the existence of unbiased estimators. We obtain a necessary form of an unbiasedly estimable parametric function $\Psi(\mu, \lambda)$ in the following theorem.

TheOREM 1. For $\exp (\mu, \lambda)$ distribution, there exists an unbiased estimator of $\Psi(\mu, \lambda)$ under time censored sampling only if

$$
\Psi(\mu, \lambda)=\text { constant } \quad \forall \mu \geq T \forall \lambda>0 .
$$


Proof. Let there exist an estimator $g(V)$ satisfying

$$
E[g(V)]=\Psi(\mu, \lambda) \quad \forall \mu, \lambda .
$$

Since for $T \leq \mu, D=0$ with probability $1,(10)$ implies $g(0,0)=\Psi(\mu, \lambda)$ for all $\mu \geq T$ and $\lambda>0$, which proves the theorem.

We immediately obtain the following result from Theorem 1 .

Theorem 2. For $\exp (\mu, \lambda)$ distribution, there does not exist any unbiased estimator of the parameters $\mu$ and $\lambda$ and also of the population mean $\mu+\lambda$ under time censored sampling.

Since $R(t)$ satisfies (9) if and only if $t \leq T$, and for $t \leq T$, a simple unbiased estimator of $R(t)$ is

$$
\widehat{R}(t)=1-D_{0} / n
$$

where $D_{0}$ is the number of items failed up to time $t$, we also have the following result.

TheOREM 3. For $\exp (\mu, \lambda)$ distribution, there exists an unbiased estimator of $R(t)$ under time censored sampling if and only if $t \leq T$.

We finally consider unbiased estimation of $R^{2}(t)$ and first prove that there does not exist any unbiased estimator for $n=1$ even for $t \leq T$.

Lemma 1. For $t \leq T$ and $n=1$, there does not exist any unbiased estimator of $R^{2}(t)$.

Proof. Let $t \leq T, n=1$ and suppose there exists an estimator $g(V)$ satisfying

$$
E[g(V)]=R^{2}(t) \quad \forall \mu \forall \lambda .
$$

As in the proof of Theorem 1, (12) implies that $g(0,0)=1$, and (12) reduces to i.e.

$$
p+\int_{\mu}^{T} g\left(1, z_{1}\right) p\left(1, z_{1}\right) d z_{1}=R^{2}(t) \quad \forall \mu<T \forall \lambda>0,
$$

$$
\frac{1}{\lambda} \int_{\mu}^{T} g\left(1, x_{(1)}\right) e^{-x_{(1)} / \lambda} d x_{(1)}= \begin{cases}e^{-\mu / \lambda}-e^{-T / \lambda}, & t \leq \mu \leq T, \lambda>0, \\ e^{-(2 t-\mu) / \lambda}-e^{-T / \lambda}, & \mu<t, \lambda>0 .\end{cases}
$$

Differentiating (13) with respect to $\mu$, for any given $\lambda$, we get

$$
g(1, \mu)= \begin{cases}1, & t \leq \mu \leq T \\ -e^{-2(t-\mu) / \lambda}, & \mu<t\end{cases}
$$

i.e.

$$
g\left(1, x_{(1)}\right)= \begin{cases}1, & t \leq x_{(1)} \leq T, \\ -e^{-2\left(t-x_{(1)}\right) / \lambda}, & x_{(1)}<t\end{cases}
$$

which is impossible as $g\left(1, x_{(1)}\right)$ cannot depend on $\lambda$. Hence, the lemma follows. 
Also for $t \leq T$ and for $n \geq 2$, an unbiased estimator of $R^{2}(t)$ is

$$
\widehat{R}^{2}(t)=\left(1-\frac{D_{0}}{n}\right)\left(1-\frac{D_{0}}{n-1}\right) .
$$

Hence, we obtain the following theorem.

TheOREM 4. For $\exp (\mu, \lambda)$ distribution, there exists an unbiased estimator of $R^{2}(t)$ under time censored sampling if and only if $t \leq T$ and $n \geq 2$.

3. Unbiased estimation of $R(t)$ and $R^{2}(t)$. In this section we discuss some unbiased estimators of $R(t)$ and $R^{2}(t)$ under time censored sampling for $t \leq T$. For unbiased estimation of $R^{2}(t)$, it is further assumed that $n \geq 2$. We note that the sufficient statistic $V$ defined in the earlier section is complete if and only if $n=1,2$ (see Bartoszewicz [1]).

For $n=1,2$ the unbiased estimator $\widehat{R}(t)$ defined in (11) is a function of the complete sufficient statistic $V$, and hence is the unique unbiased estimator of $R(t)$ based on $V$ or the UMVUE of $R(t)$. For $n>2$ and for $t<T, \widehat{R}(t)$ is not based on $V$ and can be improved upon by an unbiased estimator obtained through Rao-Blackwellization. The Rao-Blackwellized form of $\widehat{R}(t)$ is given by

$$
\widehat{R}^{*}(t)=E[\widehat{R}(t) \mid V]=1-\frac{1}{n} \sum_{k=0}^{D} k P\left(D_{0}=k \mid V\right) .
$$

An explicit expression for $P\left(D_{0}=k \mid V\right)$ is given in Bartoszewicz [1]. For $n>2, \widehat{R}^{*}(t)$ is not, however, the only unbiased estimator based on $V$. In what follows we obtain an alternative unbiased estimator of $R(t)$ based on $V$. We first prove that an estimator $\widehat{R}_{0}(t)$ defined as

$$
\widehat{R}_{0}(t)= \begin{cases}1, & X_{(1)} \geq t, \\ \frac{n-1}{n} I\left(X_{(2)}-X_{(1)}>\frac{t-X_{(1)}}{n-1}\right), & X_{(1)}<t\end{cases}
$$

is an unbiased estimator of $R(t)$ for $t \leq T$.

TheOrem 5. For $t \leq T$, the estimator $\widehat{R}_{0}(t)$ defined in $(16)$ is an unbiased estimator of $R(t)$ for $\exp (\mu, \lambda)$ distribution under time censored sampling.

Proof. For $\mu \geq t$, it is obvious that $E\left[\widehat{R}_{0}(t)\right]=R(t)$. For $\mu<t$, it can be readily verified as before that the conditional p.f. of $Y=X_{(2)}-x_{(1)}$ given $D=d(\geq 2)$ and $X_{(1)}=x_{(1)}(<t)$ is

$$
p\left(y \mid d, x_{(1)}\right)=\frac{(d-1) e^{-y / \lambda}\left[e^{-y / \lambda}-e^{-\left(T-x_{(1)}\right) / \lambda}\right]^{d-1}}{\lambda\left[1-e^{-\left(T-x_{(1)}\right) / \lambda}\right]^{d-1}}, \quad, \quad 0<y<T-x_{(1)},
$$


whence simple calculations give

$$
\begin{aligned}
\frac{n}{n-1} E\left[\widehat{R}_{0}(t) \mid\right. & \left.D=d, X_{(1)}=x_{(1)}\right] \\
& =P\left(Y>\left(t-x_{(1)}\right) /(n-1) \mid D=d, X_{(1)}=x_{(1)}\right) \\
& =\frac{\left[e^{-\left(t-x_{(1)}\right) /(n-1) \lambda}-e^{-\left(T-x_{(1)}\right) / \lambda}\right]^{d-1}}{\left[1-e^{-\left(T-x_{(1)}\right) / \lambda}\right]^{d-1}},
\end{aligned}
$$

and consequently by (6) and (18),

$$
\begin{aligned}
& E \widehat{R}_{0}(t)=p^{n}+\sum_{d=1}^{n}\left(\begin{array}{l}
n \\
d
\end{array}\right) p^{n-d} q^{d} \\
& \quad \times\left[\int_{t}^{T} p\left(x_{(1)} \mid d\right) d x_{(1)}+\int_{\mu}^{t} p\left(x_{(1)} \mid d\right) E\left[\widehat{R}_{0}(t) \mid D=d, X_{(1)}=x_{(1)}\right] d x_{(1)}\right] \\
& =p^{n}+\frac{1}{\lambda}\left[n \int_{t}^{T} e^{-n\left(x_{(1)}-\mu\right) / \lambda} d x_{(1)}+(n-1) e^{-(t-\mu) / \lambda} \int_{\mu}^{t} e^{-(n-1)\left(x_{(1)}-\mu\right) / \lambda} d x_{(1)}\right] \\
& =R(t) .
\end{aligned}
$$

This completes the proof of the theorem.

For $n>2$, the estimator $\widehat{R}_{0}(t)$ is not again a function of $V$, and an improved unbiased estimator based on $V$ is $\widehat{R}_{0}^{*}(t)=E\left[\widehat{R}_{0}(t) \mid V\right]$. The explicit form of $\widehat{R}_{0}^{*}(t)$ is derived in the following theorem.

THEOREM 6. We have

$$
\widehat{R}_{0}^{*}(t)=\left\{\begin{array}{lc}
\frac{1,}{n-1} & X_{(1)} \geq t, \\
& X_{(1)}<t, D=1, \\
\frac{n-1}{n} \frac{f_{D}\left(S_{D}-\frac{(D-1)\left(t-X_{(1)}\right)}{n-1}, T-X_{(1)}-\frac{\left(t-X_{(1)}\right)}{n-1}\right)}{f_{D}\left(S_{D}, T-X_{(1)}\right)}, \\
X_{(1)}<t, D=2, \ldots, n,
\end{array}\right.
$$

where $f_{d}(u, w)$ is defined in (8).

Proof. For $X_{(1)} \geq t$ and for $X_{(1)}<t, D=1$, (19) is obvious. Let now $d(\geq 2)$ and $x_{(1)}(<t)$ be conditionally fixed and define $Y=X_{(2)}-x_{(1)}$, $t^{*}=\left(t-x_{(1)}\right) /(n-1)$. Then

$$
\begin{aligned}
P\left(Y>t^{*} \mid D=d, X_{(1)}\right. & \left.=x_{(1)}, S_{d}=s_{d}\right) \\
& =\frac{p\left(s_{d} \mid d, x_{(1)}, Y>t^{*}\right) P\left(Y>t^{*} \mid d, x_{(1)}\right)}{p\left(s_{d} \mid d, x_{(1)}\right)}
\end{aligned}
$$


where $P\left(Y>t^{*} \mid d, x_{(1)}\right)$ is given by (18) and $p\left(s_{d} \mid d, x_{(1)}, Y>t^{*}\right)$ is the conditional p.f. of $S_{d}$ given $D=d, X_{(1)}=x_{(1)}$ and $Y>t^{*}$. It again follows as before that

$$
\begin{aligned}
p\left(s_{d} \mid d, x_{(1)}, Y>t^{*}\right) & \\
= & \frac{e^{(d-1) t^{*} / \lambda}}{\Gamma(d-1) \lambda^{d-1}\left(1-e^{-\left(T-x_{(1)}-t^{*}\right) / \lambda}\right)^{d-1}} \\
& \times e^{-s_{d} / \lambda} f_{d}\left(s_{d}-(d-1) t^{*}, T-x_{(1)}-t^{*}\right), \\
& (d-1) t^{*}<s_{d}<(d-1)\left(T-x_{(1)}\right) .
\end{aligned}
$$

By (18), (20) and (21) we get

$$
P\left(Y>t^{*} \mid D=d, X_{(1)}=x_{(1)}, S_{d}=s_{d}\right)=\frac{f_{d}\left(s_{d}-(d-1) t^{*}, T-x_{(1)}-t^{*}\right)}{f_{d}\left(s_{d}, T-x_{(1)}\right)},
$$

and this proves the theorem.

It follows similarly that for $n=2, \widehat{R}^{2}(t)$ defined in (14) is the unique unbiased estimator of $R^{2}(t)$ based on $V$ or the UMVUE. However, for $n>2$ and $t<T$, a uniformly better unbiased estimator is

$$
\widehat{R}^{2^{*}}(t)=E\left[\widehat{R}^{2}(t) \mid V\right]=\sum_{k=0}^{D}\left(1-\frac{k}{n}\right)\left(1-\frac{k}{n-1}\right) P\left(D_{0}=k \mid V\right) .
$$

Further, an alternative unbiased estimator of $R^{2}(t)$ is

$$
\widehat{R}_{0}^{2}(t)= \begin{cases}1, & X_{(1)} \geq t, \\ \frac{n-1}{n} I\left(X_{(2)}-X_{(1)}>\frac{2\left(t-X_{(1)}\right)}{n-1}\right), & X_{(1)}<t\end{cases}
$$

which yields an alternative unbiased estimator based on $V$, viz.

$$
\begin{aligned}
& \widehat{R}_{0}^{2^{*}}(t)=E\left[\widehat{R}_{0}^{2}(t) \mid V\right] \\
& \quad=\left\{\begin{array}{rc}
\frac{n-1}{n}, & X_{(1)} \geq t, \\
\frac{n-2}{n} \frac{f_{D}\left(S_{D}-\frac{2(D-1)\left(t-X_{(1)}\right)}{n-1}, T-X_{(1)}-\frac{2\left(t-X_{(1)}\right)}{n-1}\right)}{f_{D}\left(S_{D}, T-X_{(1)}\right)}, \\
X_{(1)}<t, D=2, \ldots, n .
\end{array}\right.
\end{aligned}
$$

Acknowledgements. The author is grateful to the referee for his helpful comments on an earlier draft of the paper. 


\section{References}

[1] J. Bartoszewicz, Estimation of reliability in the exponential case (II), Zastos. Mat. 14 (1975), 513-527.

[2] A. P. Basu, Estimates of reliability for some distributions useful in life testing, Tecnometrics 6 (1964), 215-219.

[3] N. L. Johnson, S. Kotz and N. Balakrishnan, Continuous Univariate Distributions, Vol. 1, 2nd ed., Wiley, New York, 1994.

[4] A. G. Laurent, Conditional distribution of order statistics and distribution of the reduced ith order statistic of the exponential model, Ann. Math. Statist. 34 (1963), $652-657$.

[5] N. R. Mann, R. E. Schafer and N. D. Singpurwalla, Methods for Statistical Analysis of Reliability and Life Data, Wiley, New York, 1974.

Department of Statistics

University of Calcutta

35, Ballygunge Circular Road

Kolkata 700019, India

E-mail: samindras@yahoo.co.in

Received on 2.6.2008;

revised version on 15.10.2008 